Pacific Journal of Mathematics

ON THE VALUE DISTRIBUTION OF FUNCTIONS
MEROMORPHIC IN THE UNIT DISK WITH A SPIRAL 


\title{
ON THE VALUE DISTRIBUTION OF FUNCTIONS MEROMORPHIC IN THE UNIT DISK WITH
}

\section{A SPIRAL ASYMPTOTIC VALUE}

\author{
JAMES R. CHOIKE
}

The object in this paper is to examine the value distribution of functions $f(z)$ nonconstant and meromorphic in the unit disk which have an asymptotic value $\alpha$, finite or infinite, along a spiral boundary path. The main result which we prove is that if $\Delta(r)$ is a component of the set of values $z$ such that $|f(z)-\alpha|<r, r>0$, which contains a boundary path on which $f(z)$ tends to $\alpha$ as $|z| \rightarrow 1$, then $f(z)$ assumes every value in $|w-\alpha|<r$ infinitely often in $\Delta(r)$ except for at most two values (if $S(r)$ is simply-connected, then there is at most one exceptional value).

1. Introduction. A boundary path $S: z=s(t), 0 \leqq t<1$, in $|z|<1$ shall be called a spiral if arg $s(t) \rightarrow+\infty$ as $t \rightarrow 1$ or arg $s(t) \rightarrow-\infty$ as $t \rightarrow 1$. We shall denote by $(S)$ the class of functions, nonconstant and meromorphic in $|z|<1$, which have an asymptotic value $\alpha$, finite or infinite, along a spiral $S$. The object in this paper is to examine the value distribution of the functions in class $(S)$. In $\S 3$ we have the main result which is a version of Picard's theorem localized to a neighborhood of a transcendental singularity for functions of class $(S)$. This result is applied in $\S 4$ to show that, in some sense, the number of direct transcendental singularities of functions of class $(S)$ cannot be too large. These results extend some earlier work of K. Noshiro [7] (see also [9, p. 163-167]).

2. Components of functions of class $(S)$. Let $f(z) \in(S)$. Then there is a complex value $\alpha$, finite or infinite, and a spiral $S: z=s(t)$, $0 \leqq t<1$, in $|z|<1$ such that $\lim _{t \rightarrow 1} f(s(t))=\alpha$. Let $r>0$ and $\omega$ be a complex number. We form the open set

$$
G=\{z|| f(z)-\omega \mid<r\}
$$

if $\omega$ is finite, and

$$
G=\{z|| f(z) \mid>r\}
$$

if $\omega$ is infinite. Since functions of class $(S)$ are of unbounded characteristic [5, p. 172], the global cluster set $C(f)$ of $f(z)$ in $|z|<1$ is total; hence, $G \neq \varnothing$. We denote by $\Delta(r)$ a nonempty open component of $G$. If $\operatorname{Fr}(\Delta(r)) \cap\{|z|=1\}=\varnothing$, where $\operatorname{Fr}(A)$ denotes the set of frontier points of the set $A$, we shall call $\Delta(r)$ a finite domain. 
If, however, $\operatorname{Fr}(\Delta(r)) \cap\{|z|=1\} \neq \varnothing$, we shall call $\Delta(r)$ an infinite domain.

By the minimum principle, each finite domain $\Delta(r)$ contains a zero of $f(z)-\omega$. Also, by Rouche's theorem, a finite domain $\Delta(r)$ contains the same number of roots, counting multiplicities, for $f(z)-\beta$ for each value $\beta,|\beta-\omega|<r$.

If $\Delta(r)$ is an infinite domain, then $\Delta(r)$ is not, in general, simplyconnected. However, an easy application of the maximum principle shows $\Delta(r)$ to be simply-connected if either $\omega \neq \infty$ and $f(z)$ is holomorphic in $|z|<1$, i.e., $f(z)$ omits $\infty$ in $|z|<1$, or $\omega=\infty$ and $f(z)$ omits 0 in $|z|<1$. An infinite domain $\Delta(r)$ with the property that the portion of its boundary $\operatorname{Fr}(\Delta(r))$ which lies within $|z|<1$ consists entirely of closed analytic curves shall be called an annular domain. An infinite domain $A(r)$ with the property that there exists a spiral $S^{*}$ in $|z|<1$ such that $\Delta(r) \leqq\{|z|<1\}-S^{*}$ shall be called a spiral domain.

THEOREM 1. If $\Delta(r)$ is an infinite domain for $f(z) \in(S)$, then $\Delta(r)$ is one of the following: (i) a spiral domain, (ii) an annular domain, or (iii) a subset of an annular domain.

Proof. Suppose $\Delta(r)$ is neither annular domain nor a spiral domain. Let $r_{1}>r$. Let $\Delta\left(r_{1}\right)$ be the component of the open set $\left\{z|| f(z)-\omega \mid<r_{1}\right\}$ such that $\Delta(r) \cong \Delta\left(r_{1}\right)$. Suppose $\Delta\left(r_{1}\right)$ is not an annular domain. Then, applying methods found in [3], we can find a boundary path $L^{\prime}$ in $|z|<1$ on which $|f(z)-\omega|=r_{1}$. Since $\Delta(r)$ is not a spiral domain, the spiral $S$ intersects $\Delta(r)$ in $1-\delta<|z|<1$ for each $\delta>0$. Thus, $|\alpha-\omega| \leqq r$, and there exists $t_{0}, 0<t_{0}<1$, such that the spiral $S^{*}: z=s(t), t_{0} \leqq t<1$, is disjoint from $L^{\prime}$. Thus, $L^{\prime}$ is also a spiral and $\Delta(r) \subseteq \Delta\left(r_{1}\right) \subseteq\{|z|<1\}-L^{\prime}$. But this implies that $\Delta(r)$ is a spiral domain, contrary to our assumption. Therefore, $\Delta\left(r_{1}\right)$ is an annular domain.

THEOREM 2. If $\Delta(r)$ is an infinite domain for $f(z) \in(S)$ and if $L$ is a boundary path in $\Delta(r)$ on which $f(z) \rightarrow \omega$ as $|z| \rightarrow 1$, then $\Delta(r)$ is either a spiral domain or an annular domain.

Proof. Recall that $\alpha$ is the asymptotic value of $f(z)$ along the spiral $S$. Suppose $\omega \neq \alpha$. Then, in this case, the boundary path $L$ must be a spiral. Suppose $\Delta(r)$ is not an annular domain. As in the proof above we apply the methods found in [3] to find a boundary path $L^{\prime}$ in $|z|<1$ on which $|f(z)-\omega|=r$. Since $L$ is a spiral, $L^{\prime}$ is a spiral and $\Delta(r) \cong\{|z|<1\}-L^{\prime}$.

Suppose $\omega=\alpha$. Then, there exists $t_{0}, 0<t_{0}<1$, such that 
$|f(s(t))-\alpha|<r$ for all $t, t_{0} \leqq t<1$. Let $S^{*}: z=s(t), t_{0}<t<1$. Clearly, either $S^{*} \leqq \Delta(r)$ or $\Delta(r) \subseteq\{|z|<1\}-S^{*}$. If $S^{*} \leqq \Delta(r)$, then we can argue as above to show that $\Delta(r)$ is a spiral domain.

Let $z=\phi(w)$ denote the inverse function of $w=f(z) \in(S)$. The damain of $z=\phi(w)$ is a Riemann surface $\Phi$. We shall write $Q\left(w ; w_{0}\right)$ to denote a functional element with center $w=w_{0}$ for $z=\phi(w)$. Let

$$
\Lambda: q(t)=Q(w ; w(t)), \quad 0 \leqq t<1,
$$

with $\lim _{t \rightarrow 1} w(t)=\omega$, be a curve on the Riemann surface $\Phi$. This curve $A$ is said to define a transcendental singularity $\Omega$ for $z=\phi(w)$ on $\Phi$, with projection $w=\omega$, if (i) for every positive number $\delta, \delta<$ 1 , the system of functional elements $Q(w ; w(t)), 0 \leqq t \leqq \delta$, defines an analytic continuation (possibly, of algebraic character), but (ii) for any functional element $Q(w ; \omega)$, rational or algebraic, with center at $w=\omega$, the system $Q(w ; w(t)), 0 \leqq t \leqq 1$, where $w(1)=\omega$, never defines an analytic continuation. A theorem due to the work of Iversen [6, p. 13] and Noshiro [7, p. 53] states that there is a one-to-one correspondence between the asymptotic paths of $w=f(z)$ and the transcendental singularities of $z=\phi(w)$, the inverse function of $w=f(z)$. In view of this result, we shall say that two asymptotic boundary paths $L_{1}$ and $L_{2}$ for $f(z) \in(S)$ are equivalent if $L_{1}$ and $L_{2}$ both correspond (in the sense of the Iversen-Noshiro theorem) to the same transcendental singularity $\Omega$ on the Riemann surface $\Phi$ of $z=\phi(w)$, and, we shall indicate this equivalence by the notation $\left[L_{1}\right]=\left[L_{2}\right]$. We refer the reader to [2] and [3] where the notions of equivalent and nonequivalent asymptotic paths are analyzed in greater detail.

THEOREM 3. If $f(z) \in(S)$ and $\Delta(r)$ is an annular domain for all $r>0$, then the inverse function $z=\phi(w)$ of $w=f(z)$ has exactly one transcendental singularity and it lies above $w=\omega$.

Proof. Suppose $z=\phi(w)$ has at least two transcendental singularities $\Omega_{1}$ and $\Omega_{2}$. Let $S_{1}$ and $S_{2}$ be the asymptotic boundary paths in $|z|<1$ for $f(z)$ which correspond to $\Omega_{1}$ and $\Omega_{2}$, respectively. Since $f(z) \in(S), S_{1}$ and $S_{2}$ are spirals. Let $r>0$. Since $\Delta(r)$ is an annular domain, $S_{1}$ and $S_{2}$ intersect $\Delta(r) \cap\{\delta<|z|<1\}$ for each $\delta, 0<\delta<1$. Since $r$ is an arbitrary positive number, we have that $\omega$ is the asymptotic value on $S_{1}$ and $S_{2}$. By [3, Theorem 1], $\left[S_{1}\right] \neq\left[S_{2}\right] \mathrm{im}$ plies that there exists $b>0$ and a boundary path $L^{\prime}$, necessarily a spiral, on which $|f(z)-\omega|=b$. But, then $\Delta\left(r_{0}\right) \subseteq\{|z|<1\}-L^{\prime}$ for $r_{0}, 0<r_{0}<b$, which contradicts our hypothesis that $\Delta\left(r_{0}\right)$ is an 
annular domain. Hence, $z=\phi(w)$ has exactly one transcendental singularity.

In [10] Valiron offers a construction of a function which shows that the converse of Theorem 3 is false. Valiron's construction is very difficult to follow and prompts the need for another approach to the construction of such a function.

3. Value distribution of functions of class $(S)$ in $\Delta(r)$. We denote by $n(\phi, a)$ the number of functional elements $Q(w ; a)$, with center $w=a$, for $z=\phi(w)$ the inverse of $f(z) \in(S)$, where an algebraic functional element is counted $k$ times if its order of ramification is $k-1$. Noshiro [7, p. 60] proved the following: Let $z=\phi_{D}(w)$ denote the branch of $z=\phi(w)$ obtained by continuing $Q(w ; a), a \in D$, inside $D$ with algebraic elements, where $D$ is an arbitrary domain of the $w$-plane. If $z=\phi_{D}(w)$ has no transcendental singularity with projection inside $D$, then $n\left(\phi_{D}, w\right)$ is a finite or infinite constant in $D$.

THEOREM 4. If $\Delta(r)$ is an annular domain for $f(z) \in(S)$ and if the transcendental singularities of $z=\phi(w)$ lying above $|w-\omega|<$ $r$ have the property that they lie above at most a finite set of points $w_{1}, w_{2}, \cdots, w_{k}$ in $|w-\omega|<r$, then every value of $|w-\omega|<r$ is assumed infinitely often by $f(z)$ in $\Delta(r)$, except possibly the values $w_{1}, w_{2}, \cdots, w_{k}$.

Proof. Let $D^{\prime}=\{|w-\omega|<r\}-\cup_{j=1}^{k}\left\{w_{j}\right\}$. Then, the branch $z=\phi_{D^{\prime}}(w)$ of $z=\phi(w)$ has no transcendental singularity with projection inside $D^{\prime}$. By Noshiro's theorem above, the function $n\left(\phi_{D^{\prime}}, w\right)$ is constant throughout $D^{\prime}$. If $\Delta(r)$ had at most finitely many holes, then, since $\Delta(r)$ is an annular domain, the global cluster set $C(f)$ of $f(z)$ would be contained in the closed disk $|w-\omega| \leqq r$. But this contradicts functions of class $(S)$ having total global cluster sets. Thus, $\Delta(r)$ has infinitely many holes. Each hole is bounded by a closed analytic curve whose image under $f(z)$ covers the circumference $|w-\omega|=r$ completely. Thus, $n\left(\phi_{D^{\prime}}, w\right)=+\infty$ throughout $D^{\prime}$ and we are done.

THEOREM 5. If $\Delta(r)$ is a spiral domain for $f(z) \in(S)$, then each value $\beta,|\beta-\omega|<r$, omitted by $f(z)$ in $\Delta(r)$ is an asymptotic value along a spiral contained in $\Delta(r)$.

Proof. Since $\Delta(r)$ is a spiral domain, there exists a spiral $S^{\prime}$ in $|z|<1$ such that $\Delta(r) \cong\{|z|<1\}-S^{\prime}$. Let $\zeta=\zeta(z)$ be a one-to-one conformal map of the simply-connected region $\{|z|<1\}-S^{\prime}$ onto 
$|\zeta|<1$ such that the prime end $P$ of $\{|z|<1\}-S^{\prime}$ whose impression $I(P)$ is $|z|=1$ corresponds to $\zeta=1$. We use $z=z(\zeta)$ to denote the inverse map of $\zeta=\zeta(z)$. Let $\Delta^{\prime}(r)$ be the image of $\Delta(r)$ in $|\zeta|<1$ under $\zeta=\zeta(z)$. Since $\Delta(r)$ is a spiral domain, we have that $1 \epsilon$ $\operatorname{Fr}\left(\Delta^{\prime}(r)\right)$.

The function $F(\zeta)=f(z(\zeta))$ is holomorphic in $\Delta^{\prime}(r)$ and continuous in $\overline{\Delta^{\prime}(r)}$, with the exception of $\zeta=1$. In fact, $|F(\zeta)-\omega|<r$ for $\zeta \in \Delta^{\prime}(r)$ and $|F(\zeta)-\omega|=r$ for $\zeta \in \operatorname{Fr}\left(\Delta^{\prime}(r)\right), \zeta \neq 1$.

Suppose $f(z)$ omits $\beta$ in $\Delta(r),|\beta-\omega|<r$. Then, $F(\zeta)$ omits $\beta$ in $\Delta^{\prime}(r)$. Let

$$
F_{2}(\zeta)=\frac{F_{1}(\zeta)-\beta^{*}}{1-\overline{\beta^{*}} F_{1}(\zeta)}
$$

where $F_{1}(\zeta)=1 / r(F(\zeta)-\omega)$ and $\beta^{*}=1 / r(\beta-\omega)$. Then, $F_{2}(\zeta)$ is holomorphic in $\Delta^{\prime}(r)$ with $\left|F_{2}(\zeta)\right|<1$ in $\Delta^{\prime}(r), \quad\left|F_{2}(\zeta)\right|=1$ for $\zeta \epsilon$ $\operatorname{Fr}\left(\Delta^{\prime}(r)\right), \zeta \neq 1$, and $F_{2}(\zeta) \neq 0$ in $\Delta^{\prime}(r)$. If $\Delta(r)$ were simply-connected, then $\Delta^{\prime}(r)$ would be simply-connected and we could, then, apply results of functions belonging to Seidel's class $(U)[8$, p. 32] to prove our theorem. Unfortunately, $\Delta(r)$ may not be simplyconnected, in general. An argument of Doob [4] helps to surmount this difficulty.

Since $F_{2}(\zeta)$ omits 0 in $\Delta^{\prime}(r), 1 / F_{2}(\zeta)$ is holomorphic in $\Delta^{\prime}(r)$. Suppose $1 / F_{2}(\zeta)$ is bounded in $\Delta^{\prime}(r)$. Then there exists a number $K>0$ such that $1 /\left|F_{2}(\zeta)\right|<K$ in $\Delta^{\prime}(r)$. Let $\sigma$ be a number such that $0<$ $\sigma<1$. Choose a definite branch of the function $(1 / 2(\zeta-1))^{\sigma}$ in $\Delta^{\prime}(r)$. This branch is holomorphic in $\Delta^{\prime}(r)$ with $|1 / 2(\zeta-1)| \leqq 1$ in $\Delta^{\prime}(r)$. The function

$$
\phi_{\sigma}(\zeta)=\frac{\left(\frac{1}{2}(\zeta-1)\right)^{\sigma}}{F_{2}(\zeta)}
$$

is holomorphic and bounded in $\Delta^{\prime}(r)$. Furthermore,

$$
\left|\phi_{o}(\zeta)\right| \leqq \frac{1}{\left|F_{2}(\zeta)\right|}=1
$$

for $\zeta \in \operatorname{Fr}\left(\Delta^{\prime}(r)\right), \zeta \neq 1$. Also,

$$
\lim _{\substack{\zeta \rightarrow 1 \\ \zeta \in \mathcal{A}^{\prime}(r)}} \phi_{\sigma}(\zeta)=0
$$

By the maximum principle, $\left|\phi_{\sigma}(\zeta)\right| \leqq 1$ for $\zeta \in \Delta^{\prime}(r)$, for every $\sigma>0$. If we let $\sigma \rightarrow 0$, we have that $\left|F_{2}(\zeta)\right| \geqq 1$ in $\Delta^{\prime}(r)$, and this is a contradiction. Thus, we have that 0 is a cluster value for $F_{2}$ at $\zeta=1$ in $\Delta^{\prime}(r)$. But, clearly, 0 does not belong to the set of boundary cluster values for $F_{2}$ at $\zeta=1$ in $\Delta^{\prime}(r)$. Since 0 is omitted by $F_{2}$ in 
$\Delta^{\prime}(r)$, by the Gross-Iversen theorem [8, p. 23-24], there exists a path $L$ in $\Delta^{\prime}(r)$ terminating at $\zeta=1$ on which $F_{2}(\zeta) \rightarrow 0$ as $|\zeta| \rightarrow 1$. Thus, on $L f(z(\zeta)) \rightarrow \beta$ as $|\zeta| \rightarrow 1$. We simply notice that the image of $L$ is a spiral lying in $\Delta(r)$ on which $f(z) \rightarrow \beta$ as $|z| \rightarrow 1$.

THEOREM 6. Let $\Delta(r)$ be an infinite domain for $f(z) \in(S)$ such that $\Delta(r)$ contains a boundary path $L$ on which $f(z) \rightarrow \omega$ as $|z| \rightarrow 1$. Let $\Delta_{\tau}=\Delta(r) \cap\{|z|<\tau\}, 0<\tau<1$, and let $A(\tau)$ denote the area of the Riemannian image of $\Delta_{\tau}$ under $w=f(z)$. Then

$$
\lim _{\tau \rightarrow 1} A(\tau)=+\infty \text {. }
$$

Proof. Suppose for all $r_{1}, 0<r_{1}<r, \Delta\left(r_{1}\right)$ is an annular domain (here it is understood, of course, that $\Delta\left(r_{1}\right)$ is that component which contains the end part of $L$ ). Then, by Theorem 3, the inverse $z=$ $\phi(w)$ has exactly one transcendental singularity which lies above $w=\omega$. By Theorem 4, every value in the disk $|w-\omega|<r$, except possibly $w=\omega$, is assumed infinitely often by $f(z)$ in $\Delta(r)$. Thus, in this case,

$$
\lim _{\tau \rightarrow 1} A(\tau)=+\infty .
$$

Let us, next, consider the case that for some $r_{0}, 0<r_{0}<r, \Delta\left(r_{0}\right)$ is a spiral domain. Let $\Delta_{\tau}^{0}=\Delta\left(r_{0}\right) \cap\{|z|<\tau\}, 0<\tau<1$, and let $A_{0}(\tau)$ denote the area of the Riemannian image of $\Delta_{\tau}^{0}$ under $f(z)$. Since $\Delta\left(r_{0}\right) \leqq \Delta(r)$, it follows that $A_{0}(\tau) \leqq A(\tau)$. In view of this, it suffices to assume that $\Delta(r)$ is a spiral domain. Since $\Delta(r) \cong\{|z|<1\}-S^{\prime}$ for some spiral $S^{\prime}$ in $|z|<1$, we again map $\Delta(r)$ onto $\Delta^{\prime}(r)$ using the one-to-one conformal $\operatorname{map} \zeta=\zeta(z)$ of $\{|z|<1\}-S^{\prime}$ onto $|\zeta|<1$ that we used in the proof of Theorem 5 . Then, the image $L^{\prime}$ of the spiral $L$ is a path in $U^{\prime}(r)$ which terminates at $\zeta=1$.

Let $\Delta^{\prime \prime}(r)$ denote the image of $\Delta^{\prime}(r)$ in the $t$-plane under the map $t=\left(\zeta-\zeta_{0}\right) /(\zeta-1)$, where $\zeta_{0}$ is the initial point of the path $L^{\prime}$. The image $L^{\prime \prime}$ of $L^{\prime}$ under this map is a path which begins at the interior point $t=0$ of $\Delta^{\prime \prime}(r)$ and terminates at the boundary point $t=\infty$ of $\Delta^{\prime \prime}(r)$. We define

$$
G(t)=f\left(z\left(\frac{t-\zeta_{0}}{t-1}\right)\right)
$$

in $\Delta^{\prime \prime}(r)$. Let $\Delta_{\tau}^{\prime \prime}=\Delta^{\prime \prime}(r) \cap\{|t|<\tau\}, 0<\tau<+\infty$. Let $A^{\prime \prime}(\tau)$ denote the area of the Riemannian image of the open set $\Delta_{\tau}^{\prime \prime}$ under $G(t)$. Since the range of $G(t)$ in $\Delta^{\prime \prime}(r)$ is identical to that of $f(z)$ in $\Delta(r)$ and since $\Delta^{\prime \prime}(r)$ is linked to $\Delta(r)$ by means of a one-to-one conformal map, it suffices to show that 


$$
\lim _{\tau \rightarrow+\infty} A^{\prime \prime}(\tau)=+\infty
$$

Let $\tau_{0}>0$ be fixed so that $|t|<\tau_{0}$ contains at least one boundary point of $\Delta^{\prime \prime}(r)$. Denote by $L_{\tau}^{\prime \prime}$ the part of the path $L^{\prime \prime}$ which runs from the last point of intersection $t_{\tau}$ of $L^{\prime \prime}$ with $|t|=\tau$, counting from $t=0$. Since $G(t) \rightarrow \omega$ on $L^{\prime \prime}$ as $|t| \rightarrow+\infty$, there exists a number $\tau_{1}, \tau_{1}>\tau_{0}>0$, such that (i) $|G(t)-\omega|<(1 / 2) r$ for all $t \in L_{\tau_{1}}^{\prime \prime}$, and (ii) for any $\tau>\tau_{1}$, if $\gamma_{\tau}$ denotes the collection of component arcs of $|t|=\tau$ which fall into $\Delta^{\prime \prime}(r)$ (there can be at most finitely many such arcs since the boundary of $\Delta^{\prime \prime}(r)$ is an analytic curve), then $\gamma_{\tau}$ contains a crosscut of $\Delta^{\prime \prime}(r)$, call it $\lambda_{\tau}$, such that the point $t_{\tau} \in \lambda_{\tau}$ and the endpoints of $\lambda_{\tau}$ lie on $\operatorname{Fr}\left(\Delta^{\prime \prime}(r)\right)$. Since the image of the arc $\lambda_{\tau}$ under $G(t)$ is a curve which starts from a point on $|w-\omega|=r$, passes through a point lying in $|w-\omega|<(1 / 2) r$, and, finally, terminates at a point on $|w-\omega|=r$, we have that the length of the image of $\lambda_{\tau}$ under $G(t)$ is greater than or equal to $r$.

Let $L(\tau)$ be the total length of the image of $\gamma_{\tau}$ under $G(t)$; let $l(\tau)$ be the total length of $\gamma_{\tau}$. Then for $t=\tau e^{i \theta}$,

$$
L(\tau)=\int_{\gamma_{\tau}}\left|G^{\prime}(t)\right| \tau d \theta
$$

By the Schwarz inequality,

$$
(L(\tau))^{2} \leqq\left(\int_{r_{\tau}}\left|G^{\prime}(t)\right|^{2} \tau d \theta\right)\left(\int_{\gamma_{\tau}} \tau d \theta\right)=l(\tau) \int_{\gamma_{\tau}}\left|G^{\prime}(t)\right|^{2} \tau d \theta
$$

Hence,

$$
\frac{(L(\tau))^{2}}{l(\tau)} \leqq \int_{\gamma_{\tau}}\left|G^{\prime}(t)\right|^{2} \tau d \theta
$$

and, for $\tau>\tau_{1}$,

$$
\int_{\tau_{1}}^{\tau} \frac{(L(\tau))^{2}}{l(\tau)} d \tau \leqq \int_{\tau_{1}}^{\tau} \int_{\tau_{\tau}}\left|G^{\prime}(t)\right|^{2} \tau d \tau d \theta \leqq A^{\prime \prime}(\tau)-A^{\prime \prime}\left(\tau_{1}\right) .
$$

Since $l(\tau) \leqq 2 \pi \tau$ and $L(\tau) \geqq r$ for all $\tau, \tau_{1} \leqq \tau \leqq+\infty$,

$$
\int_{\tau_{1}}^{\tau} \frac{(L(\tau))^{2}}{l(\tau)} d \tau \geqq \frac{r^{2}}{2 \pi} \int_{\tau_{1}}^{\tau} \frac{d \tau}{\tau} \longrightarrow+\infty
$$

as $\tau \rightarrow+\infty$. Therefore,

$$
\lim _{\tau \rightarrow+\infty} A^{\prime \prime}(\tau)=+\infty
$$

THEOREM 7. Under the hypothesis of Theorem 6, 


$$
\liminf _{t \rightarrow 1} \frac{L(\tau)}{A(\tau)}=0,
$$

where $L(\tau)$ denotes the length of the images of the collection of arcs of $|z|=\tau$ which fall into $\Delta(r)$ under $f(z)$.

Proof. By (2)

$$
\frac{(L(\tau))^{2}}{l(\tau)} \leqq \frac{d A^{\prime \prime}(\tau)}{d \tau}
$$

Hence,

$$
\frac{d \tau}{l(\tau)} \leqq \frac{d A^{\prime \prime}(\tau)}{(L(\tau))^{2}}
$$

Let $E=\left\{\tau \mid \tau>\tau_{0}, L(\tau) \geqq A^{\prime \prime}(\tau)^{1 / 2+\varepsilon}\right\}, 0<\varepsilon<1 / 2$. Since $l(\tau) \leqq 2 \pi \tau$,

$$
\frac{1}{2 \pi} \int_{E} \frac{d \tau}{\tau} \leqq \int_{E} \frac{d \tau}{l(\tau)}
$$

Thus,

$$
\begin{aligned}
\frac{1}{2 \pi} \int_{E} \frac{d \tau}{\tau} & \leqq \int_{E} \frac{d A^{\prime \prime}(\tau)}{(L(\tau))^{2}} \leqq \int_{E} \frac{d A^{\prime \prime}(\tau)}{\left(A^{\prime \prime}(\tau)^{1 / 2+\varepsilon}\right)^{2}} \\
& \leqq \int_{A^{\prime \prime}\left(\sigma_{0}\right)}^{\infty} \frac{d t}{t^{1+2 \varepsilon}}<+\infty
\end{aligned}
$$

Thus, there exists a sequence of positive numbers $\left\{\tau_{n}\right\}$ such that $\tau_{n} \rightarrow+\infty$ as $n \rightarrow+\infty$ with $\tau_{n} \notin E$ for all $n$. Therefore,

$$
\frac{L\left(\tau_{n}\right)}{A^{\prime \prime}\left(\tau_{n}\right)} \leqq \frac{A^{\prime \prime}\left(\tau_{n}\right)^{1 / 2+\varepsilon}}{A^{\prime \prime}\left(\tau_{n}\right)}=\frac{1}{A^{\prime \prime}\left(\tau_{n}\right)^{1 / 2-\varepsilon}}
$$

and, by Theorem 6, we have

$$
\liminf _{\tau \rightarrow+\infty} \frac{L(\tau)}{A^{\prime \prime}(\tau)}=0
$$

For $f(z) \in(S)$, the Riemannian image $\Phi_{r}$ of the disk $|z|<r$, $0<r<1$, under $f(z)$ is a finite covering of the Riemann sphere of diameter 1 and tangent to the $w$-plane endowed with the spherical distance as metric. The Riemann images $\Phi_{r}$ exhaust the surface $\Phi$ of the inverse $z=\phi(w)$ of $w=f(z)[8, \mathrm{p}$. 90]. Let $A(r)$ denote the spherical area of $\Phi_{r}$ and let $L(r)$ denote the spherical length of the boundary of $\Phi_{r}$.

CoROLlary. For $f(z) \in(S)$,

$$
\lim _{r \rightarrow 1} A(r)=+\infty,
$$


and

$$
\underset{r \rightarrow 1}{\liminf } \frac{L(r)}{A(r)}=0
$$

Proof. Follows immediately from Theorems 6 and 7 .

A Riemann surface $\Phi$ which satisfies condition (ii) in the above corollary is called regularly exhaustible. For a thorough discussion of regularly exhaustible surfaces and the value distribution theory connected with them we refer the reader to [9, p. 152-170]. In view of this, it is appropriate to state the following corollary.

Corollary. The Riemannian image of a function of class (S) is regularly exhaustible.

The next theorem is a Picard theorem localized to a transcendental singularity of the inverse function $z=\phi(w)$ of $f(z) \in(S)$. The idea behind the proof comes from the work of K. Noshiro [7].

THEOREM 8. Let $\Delta(r)$ be an infinite domain for $f(z) \in(S)$ such that $\Delta(r)$ contains a boundary path $L$ on which $f(z) \rightarrow \omega$ as $|z| \rightarrow 1$. Then, the function takes every value of $|w-\omega|<r$ infinitely often in $\Delta(r)$, except for at most two values. In particular, if $\Delta(r)$ is simply-connected, then $f(z)$ assumes every value of $|w-\omega|<r$ infinitely often in $\Delta(r)$ with one possible exception.

Proof. We assume, first, that $\Delta(r)$ is simply-connected. Let $\Delta^{\prime \prime}(\tau), A^{\prime \prime}(\tau), \gamma_{\tau}, L(\tau)$, and $G(t)$ be as found in the proof of Theorem 6 . Suppose that $w_{1}$ and $w_{2}$ are distinct values in $|w-\omega|<r$ such that $G(t)$ omits $w_{1}$ and $w_{2}$ in $\Delta^{\prime \prime}(r) \cap\left\{|t|>\tau_{0}\right\}$ for some $\tau_{0}>0$. Consider the open set $\Delta_{\tau}^{\prime \prime}=\Delta^{\prime \prime}(r) \cap\left\{\tau_{0}<|t|<\tau\right\}$. Since each component of $\Delta_{\tau}^{\prime \prime}$ contains at least one arc of either $\gamma_{\tau_{0}}$ or $\gamma_{\tau}$, we must have that $\Delta_{\tau}^{\prime \prime}$ consists of finitely many simply-connected components

$$
\Delta_{\tau}^{\prime \prime}(1), \Delta_{\tau}^{\prime \prime}(2), \cdots, \Delta_{:}^{\prime \prime}(m), m=m(\tau) \text {. }
$$

The Riemannian image of $\Delta_{\tau}^{\prime \prime}$ under $G(t)$ consists of $m$ simply-connected covering surfaces $\Phi_{:}^{\prime \prime}(j)$ corresponding to $\Delta_{:}^{\prime \prime}(j), j=1,2, \cdots, m$, of the base surface

$$
B=\{|w-\omega|<r\}-\left\{w_{1}, w_{2}\right\} .
$$

The Euler characteristic of $B$ is 1 [5, p. 136].

Applying Ahlfors' theorem of covering surfaces [5, p. 137] to $\Delta_{\tau}^{\prime \prime}(j)$ and $\Phi_{\tau}^{\prime \prime}(j)$, we have $S^{j} \leqq h L^{j}, j=1,2, \cdots, m$, where $S^{j}$ denotes the ratio between the area of $\Phi_{\tau}^{\prime \prime}(j)$ and the area of $B$, and $L^{j}$ 
denotes the length of the boundary of $\Phi_{\tau}^{\prime \prime}(j)$ relative to $B, h$ being a constant dependent on $B$. Thus,

$$
\sum_{j=1}^{m} S^{j} \leqq h \sum_{j=1}^{m} L^{j},
$$

and

$$
S(\tau) \leqq h\left(L(\tau)+L\left(\tau_{0}\right)\right)
$$

where $S(\tau)=A^{\prime \prime}(\tau) / \pi r^{2}$. Thus, $\left(L(\tau)+L\left(\tau_{0}\right)\right) / S(\tau) \geqq 1 / h$ for all $\tau$, $\tau>\tau_{0}$. Therefore,

$$
\lim _{: \rightarrow+\infty} \frac{L(\tau)}{A^{\prime \prime}(\tau)} \geqq \frac{\pi r^{2}}{h}>0 .
$$

But this contradicts (3). Thus, $G(t)$ in $\Delta^{\prime \prime}(r)$ and, clearly, $f(z)$ in $\Delta(r)$ assumes all values of $|w-\omega|<r$, except possibly one, infinitely many times.

Suppose $\Delta(r)$ is an arbitrary infinite domain which contains $L$; $\Delta(r)$ may not be simply-connected. Assume that there are three distinct values $w_{1}, w_{2}, w_{3}$ of $|w-\omega|<r$ which are assumed only finitely many times by $f(z)$ in $\Delta(r)$. We draw a simple closed analytic curve $L$ in $|w-\omega|<r$ which encloses $\omega, w_{1}, w_{2}$, and passes through $w_{3}$. Since $f(z) \rightarrow \omega$ on $L$ as $|z| \rightarrow 1$, we may assume that the image of $L$ under $f(z)$ lies entirely in the interior $H$ of $L$.

Let $A_{H}$ be the component of $\{z \mid f(z) \in H\}$ which contains the path $L$. Then, clearly, $\Delta_{H} \subseteq \Delta(r)$. Choose $r_{0}, 0<r_{0}<1$, such that $f(z)$ omits $w_{1}, w_{2}$, and $w_{3}$ in $\Delta(r) \cap\left\{r_{0}<|z|<1\right\}$. Let $\Delta_{H}^{*}$ be the component of $\Delta_{H} \cap\left\{r_{0}<|z|<1\right\}$ which contains the end part of $L$. Then, $\Delta_{H}^{*} \cong \Delta_{H}$, and $\Delta_{H}^{*}$ is simply-connected. Indeed, if it were not, then on the boundary of each hole $f(z)$ would assume the value $w_{3}$. But by the construction of $\Delta_{H}^{*}$ this is impossible. Also, $\Delta_{H}^{*}$ is clearly a spiral domain.

We can, now, apply the above argument to the simply-connected spiral domain $\Delta_{H}^{*}$ to show that it cannot omit two values. Thus, our theorem is proved.

THEOREM 9. Each exceptional value of Theorem 8 is an asymptotic value for $f(z)$ along a spiral contained in $\Delta(r)$.

Proof. Suppose $\beta \neq \omega$ is assumed by $f(z)$ in $\Delta(r)$ only finitely many times. Then, there exists $\delta, 0<\delta<1$, such that $f(z)$ omits $\beta$ in $\Delta(r) \cap\{\delta<|z|<1\}$. Choose $\delta_{1}, \delta<\delta_{1}<1$, so that $f(z) \neq \beta$ on $|z|=\delta_{1}$. Let

$$
\varepsilon=\min |f(z)-\beta| \text { for }|z|=\delta_{1},
$$


and, let $\rho=1 / 4 \min (\varepsilon, r-|\beta-\omega|,|\beta-\omega|)$. Clearly, $\rho>0$. By Theorem 8, there exists a value $z_{0}$ such that $z_{0} \in \Delta(r) \cap\left\{\delta_{1}<|z|<1\right\}$ and $\left|f\left(z_{0}\right)-\beta\right|<\rho$. Let $\Delta_{\beta}(\rho)$ be that component of $\{z \| f(z)-\beta \mid<\rho\}$ which contains $z_{0}$. By the choice of $\rho$, we have $\Delta_{\beta}(\rho) \subseteq\left\{\delta_{1}<|z|<1\right\}$ and $\Delta_{\beta}(\rho) \subseteq \Delta(r)$. Since $f(z)$ omits $\beta$ in $\Delta_{\beta}(\rho)$, we have that $\Delta_{\beta}(\rho)$ is an infinite domain. We, also, point out that the end part of the spiral on which $f(z) \rightarrow \omega$ as $|z| \rightarrow 1$ is disjoint from $\Delta_{\beta}(\rho)$. Thus, $\Delta_{\beta}(\rho)$ is a spiral domain in which $\beta$ is an omitted value of $f(z)$. By Theorem $5, \beta$ is an asymptotic value along a spiral contained in $\Delta_{\beta}(\rho)$.

4. Direct transcendental singularities. Let $f(z) \in(S)$ and $\Delta(r)$ be an infinite domain in $|z|<1$ such that $\Delta(r)$ contains a boundary path $L$ on which $f(z) \rightarrow \omega$. If $f(z)$ omits $\omega$ in $\Delta(r)$ for $r>0$ sufficiently small, then the transcendental singularity $\Omega$ of $z=\phi(w)$ which corresponds to $L$ is said to be a direct transcendental singularity.

THEOREM 10. Let $f(z) \in(S)$ and let $z=\phi(w)$ be its inverse function. Then, the set of values $\omega$ in the $w$-plane which are projections of direct transcendental singularities of $z=\phi(w)$ is at most countable.

Proof. Let $\left\{\omega_{n}\right\}$ be the rational points in the $w$-plane and let $\left\{r_{n}\right\}$ be the rationals of the interval $(0,1)$. Let $G_{n}=\left\{z|| f(z)-\omega_{n} \mid<r_{n}\right\}$. We set $H=\cup_{n=1}^{\infty} H_{n}$, where $H_{n}$ is the set of points of $\left|w-\omega_{n}\right|<r_{n}$ which are not covered by the image of at least one component of $G_{n}$ under $f(z)$. By Theorem $9, H_{n}$ is at most countable, and, hence, so is $H$.

Suppose $f(z) \rightarrow \omega$ on a spiral $S$ and $S$ corresponds to a direct transcendental singularity for $z=\phi(w)$. Then, there exists $r>0$ such that $S \subset \Delta(r)$ and $f(z)$ omits $\omega$ in $\Delta(r)$. But there exists an integer $n$ such that a component $\Delta_{n}$ of $G_{n}$ is contained in $\Delta(r)$ with $\left|\omega-\omega_{n}\right|<r_{n}$. Therefore, $\omega \in H_{n}$ and the theorem is proven.

We, next, present an example of a holomorphic function $f(z) \in(S)$ such that its inverse function has uncountably many transcendental singularities above $w=\infty$, and we note that since $f(z)$ is holomorphic these are direct transcendental singularities. This example places the last result in clearer perspective.

Let $A$ be the extended complex plane and let $M$ be the Cantor set on the interval $0 \leqq \theta \leqq 2 \pi$ with $\left\{I_{n}\right\}$ denoting the sequence of the open middle-third intervals of $0 \leqq \theta \leqq 2 \pi$ which are deleted to construct $M$. The order of the sequence $\left\{I_{n}\right\}$ is as follows: 


$$
\begin{aligned}
& I_{1}=\left(\frac{2 \pi}{3}, \frac{4 \pi}{3}\right), \quad I_{2}=\left(\frac{2 \pi}{9}, \frac{4 \pi}{9}\right), \\
& I_{3}=\left(\frac{14 \pi}{9}, \frac{16 \pi}{9}\right), \quad I_{4}=\left(\frac{2 \pi}{27}, \frac{4 \pi}{27}\right), \cdots .
\end{aligned}
$$

Let $\left\{J_{n}\right\}$ be the sequence of open intervals of the sequence of open sets $[0,2 \pi]-\bar{I}_{1},[0,2 \pi]-\bar{I}_{1} \cup I_{2}, \cdots$, with the ordering

$$
\begin{aligned}
& J_{1}=\left(0, \frac{2 \pi}{3}\right), \quad J_{2}=\left(\frac{4 \pi}{3}, 2 \pi\right), \quad J_{3}=\left(0, \frac{2 \pi}{9}\right), \\
& J_{4}=\left(\frac{4 \pi}{9}, \frac{2 \pi}{3}\right), \quad J_{5}=\left(\frac{4 \pi}{3}, \frac{14 \pi}{9}\right), \cdots .
\end{aligned}
$$

Since $A$ is an analytic set and $M$ is a closed nowhere dense set we can apply a theorem of Bagemihl and Seidel [1, p. 198-199] to claim the existence of a function $f(z)$, holomorphic in $|z|<1$, with the following properties:

(i) for every $\theta \in M, \lim _{r \rightarrow 1} f\left(\mathrm{re}^{i(\theta+1 /(1-r))}\right)=w_{\theta}$ exists (possibly infinite);

(ii) if $I$ is any subinterval of $0 \leqq \theta \leqq 2 \pi$ such that $I \cap M \neq \varnothing$, then $A=\left\{w_{\theta} \mid \theta \in I \cap M\right\}$, and for every $a \in A$, there are uncountably many values of $\theta \in I \cap M$ for which $w_{\theta}=a$.

By (ii) every value of the extended complex plane is an asymptotic value on uncountably many spiral paths $\left.S_{\theta}: z=s_{\theta}(r)=\mathrm{re}^{i(\theta+1 /(1-r))}\right)$, $0 \leqq r<1$, for $\theta \in M$. Let $\theta_{1}, \theta_{2} \in M, \theta_{1}<\theta_{2}$ such that $f(z) \rightarrow \omega$ on $S_{\theta_{1}}$ and $S_{\theta_{2}}$ as $|z| \rightarrow 1$. We can find two intervals $I_{n_{1}}$ and $I_{n_{2}}$ of $\left\{I_{n}\right\}$ such that $I_{n_{1}} \subseteq\left(\theta_{1}, \theta_{2}\right)$ and $I_{n_{2}} \leqq[0,2 \pi]-\left[\theta_{1}, \theta_{2}\right]$. Thus, for $n_{1}^{\prime}, n_{2}^{\prime}$ sufficiently large, there exist intervals $J_{n_{1}^{\prime}}$ abutting $I_{n_{1}}$ and $J_{n_{2}^{\prime}}$ abutting $I_{n_{2}}$ such that $J_{n_{1}^{\prime}} \leqq\left(\theta_{1}, \theta_{2}\right)$ and $J_{n_{2}^{\prime}} \leqq[0,2 \pi]-\left[\theta_{1}, \theta_{2}\right]$. But since $J_{n_{1}^{\prime}} \cap M \neq \varnothing$ and $J_{n_{2}^{\prime}} \cap M \neq \varnothing$, by (ii), there exist spirals separating $S_{\theta_{1}}$ and $S_{\theta_{2}}$ on which $f(z)$ has asymptotic values different from $w=\omega$. Thus $\left[S_{\theta_{1}}\right] \neq\left[S_{\theta_{2}}\right]$. Since $\omega$ is an arbitrary value of the extended complex plane, we see that the inverse $z=\phi(w)$ has uncountably many transcendental singularities above every value of the extended $w$-plane. In particular, since $f(z)$ omits $w=\infty, z=$ $\phi(w)$ has uncountably many direct transcendental singularities above $w=\infty$.

\section{REFERENCES}

1. F. Bagemihl and W. Seidel, Some boundary properties of analytic functions, Math. Z., 61 (1954), 186-199.

2. J. R. Choike, On the asymptotic boundary paths of analytic functions, J. reine angew. Math., 264 (1974), 29-39.

3. - On the asymptotic boundary behavior of functions analytic in the unit disk, (submitted for publication). 
4. J. L. Doob, On a theorem of Gross and Iversen, Ann. of Math., (2) 33 (1932), 753-757.

5. W. K. Hayman, Meromorphic Functions, Oxford Mathematical Monographs, Oxford, 1964.

6. F. Iversen, Recherches sur les fonctions inverses des fonctions méromorphes, Thèse, Helsingfors, 1914.

7. K. Noshiro, On the singularities of analytic functions, Japan J. Math., 17 (1940), $37-96$.

8. Cluster Sets, Springer-Verlag, Berlin-Göttingen-Heidelberg, 1960.

9. L. Sario and K. Noshiro, Value Distribution Theory, D. Van Nostrand Co., Inc., New York, 1966.

10. G. Valiron, Sur les singularités de certaines fonctions holomorphes et de leur inverses, J. Math. Pures et Appl., 15 (1936), 423-435.

Received October 22, 1974.

OKLahoma State University 



\section{PACIFIC JOURNAL OF MATHEMATICS}

\section{EDITORS}

RICHARD ARENS (Managing Editor)

University of California

Los Angeles, California 90024

\section{J. DugunduI}

Department of Mathematics University of Southern California Los Angeles, California 90007

D. Gilbarg and J. Milgram

Stanford University

Stanford, California 94305

\author{
R. A. Beaumont \\ University of Washington \\ Seattle, Washington 98105
}

\section{ASSOCIATE EDITORS}
B. H. NeUmanN
F. WOLF
K. YosHIDA

\section{SUPPORTING INSTITUTIONS}

\author{
UNIVERSITY OF BRITISH COLUMBIA \\ CALIFORNIA INSTITUTE OF TECHNOLOGY \\ UNIVERSITY OF CALIFORNIA \\ MONTANA STATE UNIVERSITY \\ UNIVERSITY OF NEVADA \\ NEW MEXICO STATE UNIVERSITY \\ OREGON STATE UNIVERSITY \\ UNIVERSITY OF OREGON \\ OSAKA UNIVERSITY
}

\author{
UNIVERSITY OF SOUTHERN CALIFORNIA \\ STANFORD UNIVERSITY \\ UNIVERSITY OF HAWAII \\ UNIVERSITY OF TOKYO \\ UNIVERSITY OF UTAH \\ WASHINGTON STATE UNIVERSITY \\ UNIVERSITY OF WASHINGTON \\ AMERICAN MATHEMATICAL SOCIETY
}

The Supporting Institutions listed above contribute to the cost of publication of this Journal, but they are not owners or publishers and have no responsibility for its content or policies.

Mathematical papers intended for publication in the Pacific Journal of Mathematics should be in typed form or offset-reproduced, (not dittoed), double spaced with large margins. Please do not use built up fractions in the text of your manuscript. You may however, use them in the displayed equations. Underline Greek letters in red, German in green, and script in blue. The first paragraph or two must be capable of being used separately as a synopsis of the entire paper. Items of the bibliography should not be cited there unless absolutely necessary, in which case they must be identified by author and Journal, rather than by item number. Manuscripts, in triplicate, may be sent to any one of the editors. Please classify according to the scheme of Math. Reviews, Index to Vol. 39. All other communications should be addressed to the managing editor, or Elaine Barth, University of California, Los Angeles, California, 90024.

The Pacific Journal of Mathematics expects the author's institution to pay page charges, and reserves the right to delay publication for nonpayment of charges in case of financial emergency.

100 reprints are provided free for each article, only if page charges have been substantially paid. Additional copies may be obtained at cost in multiples of 50 .

The Pacific Journal of Mathematics is issued monthly as of January 1966. Regular subscription rate: $\$ 72.00$ a year (6 Vols., 12 issues). Special rate: $\$ 36.00$ a year to individual members of supporting institutions.

Subscriptions, orders for back numbers, and changes of address should be sent to Pacific Journal of Mathematics, 103 Highland Boulevard, Berkeley, California, 94708.

PUBLISHED BY PACIFIC JOURNAL OF MATHEMATICS, A NON-PROFIT CORPORATION

Printed at Kokusai Bunken Insatsusha (International Academic Printing Co., Ltd.), 8-8, 3-chome, Takadanobaba, Shinjuku-ku, Tokyo 160, Japan. 


\section{Pacific Journal of Mathematics}

Patricia Andresen and Marvin David Marcus, Weyl's inequality and

quadratic forms on the Grassmannian .......................

George Bachman and Alan Sultan, Regular lattice measures: mappings and

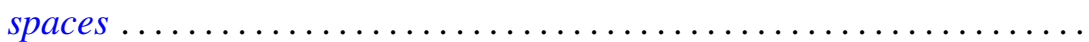

David Geoffrey Cantor, On certain algebraic integers and approximation by rational functions with integral coefficients ...................

James Richard Choike, On the value distribution of functions meromorphic in the unit disk with a spiral asymptotic value ..................

David Earl Dobbs, Divided rings and going-down................ 353

Mark Finkelstein and Robert James Whitley, Integrals of continuous

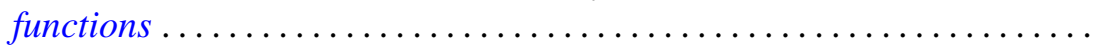

Ronald Owen Fulp and Joe Alton Marlin, Integrals of foliations on manifolds with a generalized symplectic structure ...............

Cheong Seng Hoo, Principal and induced fibrations .................

Wu-Chung Hsiang and Richard W. Sharpe, Parametrized surgery and

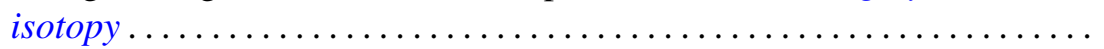

Surender Kumar Jain, Surjeet Singh and Robin Gregory Symonds, Rings whose proper cyclic modules are quasi-injective .................

Pushpa Juneja, On extreme points of the joint numerical range of commuting normal operators...

Athanassios G. Kartsatos, Nth order oscillations with middle terms of order $N-2$.

John Keith Luedeman, The generalized translational hull of a

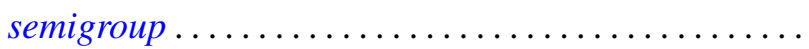

Louis Jackson Ratliff, Jr., The altitude formula and DVR's ...

Ralph Gordon Stanton, C. Sudler and Hugh C. Williams, An upper bound for the period of the simple continued fraction for $\sqrt{D}$...

David Westreich, Global analysis and periodic solutions of second order systems of nonlinear differential equations...

David Lee Armacost, Correction to: "Compactly cogenerated LCA

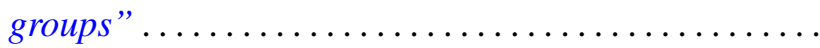

Jerry Malzan, Corrections to: "On groups with a single involution" .

David Westreich, Correction to: "Bifurcation of operator equations with unbounded linearized part" ...................... 